\title{
Berezin Quantization of Gaussian Functions Depending by a Quantum and Compression Parameter*
}

\author{
Simone Camosso \\ I.I.S. “G. Vallauri”, Fossano 12045 (CN), Piedmont, Italy \\ Email: r.camosso@alice.it.
}

How to cite this paper: Camosso, S. (2019) Berezin Quantization of Gaussian Functions Depending by a Quantum and Compression Parameter. Journal of Quantum Information Science, 9, 15-21. https://doi.org/10.4236/jqis.2019.91002

Received: February 4, 2019

Accepted: March 5, 2019

Published: March 8, 2019

Copyright $\odot 2019$ by author(s) and Scientific Research Publishing Inc. This work is licensed under the Creative Commons Attribution International License (CC BY 4.0).

http://creativecommons.org/licenses/by/4.0/

\begin{abstract}
The aim of this work is to study the Berezin quantization of a Gaussian state. The result is another Gaussian state that depends on a quantum parameter $\alpha$, which describes the relationship between the classical and quantum vision. The compression parameter $\lambda>0$ is associated to the harmonic oscillator semigroup.
\end{abstract}

\section{Keywords}

Berezin Quantization, Berezin Transform, Gaussian Functions, Quantum Harmonic Oscillator, Heisenberg Uncertainty Principle

\section{Introduction}

This paper is devoted to the study of the quantization of Gaussian states. Let us consider a function $f(x, p)$, with $(x, p) \in \mathbb{R}^{2 n}$, denoted by $B_{\alpha}(f)$ as its Berezin quantization. The choice of the Berezin quantization is due to the fact that we will consider Gaussian functions on $\mathbb{C}^{n}$ instead $\mathbb{R}^{2 n}$ and, for this reason, a good scheme of quantization of $\mathbb{C}^{n}$ is the Berezin quantization. It is well known that this scheme of quantization in comparison with the Weyl quantization presents "a few problems": for example, it doesn't preserve polynomial relations, the product rules are more complicated than Weyl quantization and the equivalent of Eherenfest theorem doesn't hold. Indeed, this scheme of quantization is rarely used to describe the system dynamics. On the other hand, it leads naturally to the definition of a vacuum state $\psi_{0}$ that is usually a Gaussian function.

*These notes on the Berezin quantization have been written when the author was a teacher at Liceo "G. B. Bodoni". 
In the first two sections we will review basic notions on the Berezin quantization of $\mathbb{C}^{n}$ and the quantum harmonic oscillator [1]-[7]. The other sections are devoted to the proofs of the following results.

Theorem 1.1 In the setting of the Berezin quantization of $\left(\mathbb{C}^{n},\langle\cdot, \cdot\rangle\right)$ we have that the quantization of the complex Gaussian $f(z)=C \mathrm{e}^{-\frac{\lambda}{4}(z+\bar{z})^{2}}$, with $C=\frac{\sqrt{\mathrm{e}}}{2^{n}}$, is given by:

$$
B_{\alpha}(f)=C^{\prime}\left(\frac{\alpha}{\alpha+\lambda}\right)^{\frac{n}{2}} \mathrm{e}^{-\frac{\alpha \lambda}{4(\alpha+\lambda)}(z+\bar{z})^{2}}
$$

where $\alpha$ is a quantum parameter, $C^{\prime}=\frac{\sqrt{\mathrm{e}}}{2^{n}}, \quad z \in \mathbb{C}^{n}$ and $\lambda>0$.

It would be desirable to evaluate the trace of the previous Berezin transform. Unfortunately, following the definition of [8] for the trace of the Berezin transform, we find an infinite value. As corollary, we can consider instead the classical trace another kind of trace deriving from the usual inner product on the space $L^{2}(\Omega, \rho)$. In this case we have a "modified" version that is the trace of the squared Berezin symbol.

$$
\text { Corollary 1.2 Let } \begin{aligned}
& \overline{B_{\alpha}(f)}=\frac{B_{\alpha}(f)}{\sqrt{C^{\prime}}\left(\frac{\alpha}{\alpha+\lambda}\right)^{\frac{n}{4}}} \text {, then we have that: } \\
& \operatorname{Tr}\left({\overline{B_{\alpha}(f)^{2}}}^{2}=\left(\frac{\alpha}{\alpha+3 \lambda}\right)^{\frac{n}{2}} .\right.
\end{aligned}
$$

We observe that the selected Gaussian function $f(z)$ depends on a "compression factor" $\lambda>0$ and that $\alpha$ is a quantum parameter that corresponds to the inverse of the Planck constant $h$. We observe that when $\alpha \rightarrow+\infty$ the trace tends to 1 . Moreover, when $\alpha=\lambda=1$ we have $\frac{1}{2^{n}}$. This can be interpreted as an index of the purity of the state. The next result I will present is inspired by the work of [9] and [10], it corresponds to a "generalized" version of the Heisenberg principle in one dimension.

Theorem 1.3 In the setting of the Berezin quantization of $\mathbb{C}$, if $\lambda>0$ then

$$
\sigma_{\psi_{\lambda}}^{2}(x) \sigma_{\psi_{\lambda}}^{2}(p)=\frac{1}{4}\left(-i[x, p] \psi_{\lambda}, \psi_{\lambda}\right)^{2},
$$

where $\psi_{\lambda}=C^{\prime}\left(\frac{\alpha}{\alpha+\lambda}\right)^{\frac{n}{2}} \mathrm{e}^{-\frac{\alpha \lambda}{4(\alpha+\lambda)}(z+\bar{z})^{2}}$ is the quantized Gaussian and $(\cdot, \cdot)$ is the inner product of elements of $L^{2}(\mathbb{R})$.

In this theorem the quantum parameter has been fixed to 1 as it is convention with the natural units.

\section{The Berezin Quantization}

Let $\Omega$ be a domain in $\mathbb{C}^{n}$ with the usual inner product 
$\langle z, w\rangle=z \cdot \bar{w}=\sum_{j=1}^{n} z_{j} \overline{w_{j}}$. Let $\rho>0$ be a weight function on $\Omega$ and $L^{2}(\Omega, \rho)$ be the space of square integrable functions respect $\rho$. Let $L_{\mathrm{hol}}^{2}(\Omega, \rho)$ be the subspace of square integrable holomorphic functions respect to $\rho$. This space is also called the "weighted Bergmann space" and has a reproducing kernel $K_{\rho}(z, w)$. Let us assume $K_{\rho}(z, z)>0$ for all $z$, we define the "Berezin transform" of $f \in L^{\infty}(\Omega)$ the following integral operator:

$$
B_{\rho} f(z)=\frac{1}{K_{\rho}(z, z)} \int_{\Omega} f(w)\left|K_{\rho}(z, w)\right|^{2} \rho(w) \mathrm{d} w \mathrm{~d} \bar{w} .
$$

The Berezin transform is an important tool in the contest of Berezin quantization, especially its asymptotic behaviour with the appropriate weights $\rho$. As showed in [2] the construction of the Berezin quantization reduces to constructing a family of weights for which the associated Berezin transform $B_{\rho}$ has an asymptotic expansion:

$$
B_{\rho_{\alpha}}=Q_{0}+\frac{1}{\alpha} Q_{1}+\frac{1}{\alpha^{2}} Q_{2}+\cdots
$$

where $\alpha=\frac{1}{h}$ is the "formal parameter" that when $h \rightarrow 0$ we have $\alpha \rightarrow+\infty$, $Q_{0}$ is the identity operator and $Q_{j}=\sum_{\beta, \gamma \in \mathbb{N}^{n}} c_{j \beta \gamma} \partial^{\beta} \bar{\partial}^{\gamma}(f)$ are differential operators with $\beta, \gamma$ multi-indices. From $Q_{j}$ it is possible to define the bidifferential operators $C_{j}(f, g)=\sum_{\beta, \gamma \in \mathbb{N}^{n}} c_{j \beta \gamma} \partial^{\beta}(f) \bar{\partial}^{\gamma}(g)$, and the star-product:

$$
f \star g=\sum_{j=0}^{+\infty} \frac{1}{\alpha^{j}} C_{j}(f, g) .
$$

If the condition $C_{1}(f, g)-C_{1}(g, f)=\frac{i}{2 \pi}\{f, g\}$ it's valid then the star product coincides with the Berezin star-product and this provides a Berezin quantization. Here $\{\cdot, \cdot\}$ is the Poisson bracket on $\mathbb{C}^{n}$ and $f, g \in C^{\infty}(\Omega)$ are quantum observables.

The proof of this assertion and many details on the Berezin quantization of $\mathbb{C}^{n}$ can be found in [2]. For the Berezin quantization of general function spaces the reader can consult [3].

For our purpose we consider the Berezin quantization of $\mathbb{C}^{n}$ with the weighted Bergmann space as function space. In this case $\rho(z)=\left(\frac{\alpha}{\pi}\right)^{n} \mathrm{e}^{-\alpha z \cdot \bar{z}}$, $\alpha$ will be the quantum parameter that tends to infinity and $K_{\rho}(z, w)=\mathrm{e}^{\alpha z \cdot \bar{w}}$.

\section{The Quantum Harmonic Oscillator}

We consider a slightly modified version of the quantum harmonic oscillator in $\mathbb{C}^{n}$. Let $H=\hat{x}^{2}+\hat{p}^{2}=\sum_{j=1}^{n} \hat{x}_{j}^{2}+\hat{p}_{j}^{2}$ be the Hamiltonian operator where $\hat{x}_{j}=x_{j}$ and $\hat{p}_{j}=-i \partial_{x_{j}}$ are the usual quantum operators that satisfy the following commutation relations:

$$
\left[x_{j}, p_{k}\right]=i h \delta_{j k},\left[x_{j}, x_{k}\right]=\left[p_{j}, p_{k}\right]=0,
$$


for every $j, k=1, \cdots, n$ and where $h$ is the Plank constant. We define the operators:

$$
\hat{z}_{j}=\frac{\hat{x}_{j}+i \hat{p}_{j}}{\sqrt{2}}, \hat{\bar{z}}_{j}=\frac{\hat{x}_{j}-i \hat{p}_{j}}{\sqrt{2}}
$$

for every $j=1, \cdots, n$. The operators $\hat{z}, \hat{\bar{z}}$ are called respectively the annihilation and creation operator. In this notation the Hamiltonian assume the following form $H=\sum_{k=1}^{n} 2 \hat{\bar{z}}_{k} \hat{z}_{k}+h$. It is possible to prove that the ground state corresponding to the energy level $E_{0}=n h$ is given by the Gaussian:

$$
\psi_{0}=\mathrm{e}^{-\frac{x^{2}}{2}},
$$

where $x^{2}=\sum_{k=1}^{n} x_{k}^{2}$. In general we have eigenvalues of energy in this even form:

$$
E_{j}=n \cdot(2 j+h),
$$

with eigenfunctions given by $\psi_{j}=\prod_{k=1}^{n} x_{k}^{j} \mathrm{e}^{-\frac{x^{2}}{2}}$.

\section{Proof of the Theorem 1.1}

Proof. The Berezin transform of $\mathrm{e}^{-\frac{\lambda}{4}(z+\bar{z})^{2}}$ with parameter $\alpha$ is:

$$
B_{\rho}(f)=\left(\frac{\alpha}{\pi}\right)^{n} \mathrm{e}^{-\alpha\langle w, w\rangle} \int_{\mathbb{C}^{n}} \mathrm{e}^{-\frac{\lambda}{4}(z+\bar{z})^{2}}\left|\mathrm{e}^{\alpha\langle w, z\rangle}\right|^{2} \mathrm{e}^{-\alpha\langle z, z\rangle} \mathrm{d} z \mathrm{~d} \bar{z} .
$$

We remember the definition of the complex inner product $\langle w, z\rangle=w \cdot \bar{z}=\sum_{j=1}^{n} w_{j} \overline{z_{j}}$ and, after an algebraic semplification, we have that:

$$
B_{\rho}(f)=\left(\frac{\alpha}{\pi}\right)^{n} \mathrm{e}^{-\alpha w \cdot \bar{w}} \int_{\mathbb{C}^{n}} \mathrm{e}^{-\frac{\lambda}{4}(z+\bar{z})^{2}+\alpha w \bar{z}+\alpha \bar{w} z-\alpha \bar{z} \bar{z}} \mathrm{~d} z \mathrm{~d} \bar{z} .
$$

This is a complex-Gauss integral depending by a quantum parameter $\alpha$ and the positive parameter $\lambda$. A simple way to solve the integral (11) consists to transform the complex integral in a real integral using the identification $\mathbb{C}^{n} \equiv \mathbb{R}^{2 n}$. This gives the product of two real Gauss integrals:

$$
\begin{aligned}
B_{\rho}(f)= & \left(\frac{\alpha}{\pi}\right)^{n} \mathrm{e}^{-\frac{\alpha}{2}\left(x^{\prime 2}+p^{\prime 2}+\frac{1}{\alpha}\right)-\frac{1}{2}} \int_{\mathbb{R}^{n}} \mathrm{e}^{-\left(\sqrt{\frac{\lambda+\alpha}{2}} x-\frac{\alpha}{2 \sqrt{\frac{\lambda+\alpha}{2}}} x^{\prime}\right)+\frac{\alpha^{2}}{2(\lambda+\alpha)} x^{\prime 2}} \mathrm{~d} x \\
& \cdot \int_{\mathbb{R}^{n}} \mathrm{e}^{-\frac{\alpha}{2}\left(p^{2}-2 p p^{\prime}+p^{\prime 2}\right)+\frac{\alpha}{2} p^{\prime 2}} \mathrm{~d} p .
\end{aligned}
$$

where $z=\frac{x+i p}{\sqrt{2}}, \bar{z}=\frac{x-i p}{\sqrt{2}}, w=\frac{x^{\prime}+i p^{\prime}}{\sqrt{2}}, \bar{w}=\frac{x^{\prime}-i p^{\prime}}{\sqrt{2}}$ and

$\left[x, x^{\prime}\right]=\left[p, p^{\prime}\right]=0,[x, p]=\left[x^{\prime}, p^{\prime}\right]=i h$ are the canonical relations. Adjusting the exponents of the two integrals we get two Gauss integrals that can be evaluated:

$$
B_{\rho}(f)=\left(\frac{\alpha}{\pi}\right)^{n} \mathrm{e}^{-\frac{\alpha}{2}\left(x^{\prime 2}+p^{\prime 2}-\frac{1}{\alpha}\right)-\frac{1}{2}+\frac{\alpha^{2}}{2(\lambda+\alpha)} x^{\prime 2}+\frac{\alpha}{2} p^{\prime 2}} \cdot \frac{\pi^{\frac{n}{2}} 2^{\frac{n}{2}}}{(\lambda+\alpha)^{\frac{n}{2}}} \cdot \frac{\pi^{\frac{n}{2}} 2^{\frac{n}{2}}}{\alpha^{\frac{n}{2}}} .
$$


Thus we find that the initial integral is equal to $2^{n}\left(\frac{\alpha}{\alpha+\lambda}\right)^{\frac{n}{2}} \mathrm{e}^{-\frac{\alpha \lambda}{2(\alpha+\lambda)} x^{x^{2}-1}}$

Observation 4.1 We observe that when $\alpha \rightarrow+\infty$ the complex quantized Gaussian $C^{\prime}\left(\frac{\alpha}{\alpha+\lambda}\right)^{\frac{n}{2}} \mathrm{e}^{-\frac{\alpha \lambda}{4(\alpha+\lambda)}(z+\bar{z})^{2}}$ tends to the classical complex Gaussian $C^{\prime} \mathrm{e}^{-\frac{\lambda}{4}(z+\bar{z})^{2}}$

Observation 4.2 We can rewrite the $\left(\frac{\alpha}{\alpha+\lambda}\right)^{\frac{n}{2}} \mathrm{e}^{-\frac{\alpha \lambda}{4(\alpha+\lambda)}(1+\bar{z})^{2}}$ as

$$
\left[\frac{1}{\left(1+\frac{\lambda}{\alpha}\right)^{\frac{n}{2}}} \mathrm{e}^{-\frac{\lambda^{2}}{4 \alpha\left(1+\frac{\lambda}{\alpha}\right)^{(z+\bar{z})^{2}}}}\right] \mathrm{e}^{-\frac{\lambda}{4}(z+\bar{z})^{2}}
$$

and Taylor expand the square brackets:

$$
\left[1+\frac{\lambda^{2}}{4 \alpha}(z+\bar{z})^{2}-\frac{n}{2} \cdot \frac{\lambda}{\alpha}+\cdots\right] \mathrm{e}^{-\frac{\lambda}{4}(z+\bar{z})^{2}} .
$$

If we not consider the term $\frac{\sqrt{\mathrm{e}}}{2^{n}}$, this is exactly the heat solution operator $B \mathrm{e}^{-\frac{\lambda}{4}(z+\bar{z})^{2}}=\mathrm{e}^{\frac{\Delta}{4 \alpha}} \mathrm{e}^{-\frac{\lambda}{4}(z+\bar{z})^{2}}$ according to [2], where $\Delta$ is the complex Laplacian on $\mathbb{C}^{n}$ given by $\Delta=4 \sum_{j=1}^{n} \partial_{w_{j}} \partial_{\overline{w_{j}}}$.

\section{Proof of the Corollary 1.2}

By the modified version of the trace ("that is a sort of a trace of a square") we have that:

$$
\operatorname{Tr}\left(B_{\alpha}(f)^{2}\right)=\left(\frac{\alpha}{\pi}\right)^{n} \int_{\mathbb{C}^{n}} B_{\alpha}(f)(z)^{2} \mathrm{e}^{-\alpha z \cdot \bar{z}} \mathrm{~d} z \mathrm{~d} \bar{z}
$$

Thus we must to evaluate the integral:

$$
\operatorname{Tr}\left(B_{\alpha}(f)^{2}\right)=\left(\frac{\alpha}{\pi}\right)^{n} \int_{\mathbb{C}^{n}} C^{\prime 2}\left(\frac{\alpha}{\alpha+\lambda}\right)^{n} \mathrm{e}^{-\frac{\alpha \lambda}{2(\alpha+\lambda)}(z+\bar{z})^{2}} \mathrm{e}^{-\alpha z \cdot \bar{z}} \mathrm{~d} z \mathrm{~d} \bar{z} .
$$

Using the canonical relations, this can be written as:

$$
\operatorname{Tr}\left(B_{\alpha}(f)^{2}\right)=\frac{e}{2^{2 n}}\left(\frac{\alpha}{\pi}\right)^{n}\left(\frac{\alpha}{\alpha+\lambda}\right)^{n} \int_{\mathbb{R}^{n}} \mathrm{e}^{\frac{-3 \alpha \lambda-\alpha^{2}}{2(\alpha+\lambda)} x^{2}} \mathrm{~d} x \cdot \int_{\mathbb{R}^{n}} \mathrm{e}^{-\frac{\alpha}{2} p^{2}-\frac{1}{2}} \mathrm{~d} p .
$$

After the evaluation the Gauss integrals we find that the initial trace is equal to $C^{\prime}\left(\frac{\alpha}{\alpha+\lambda}\right)^{\frac{n}{2}} \cdot\left(\frac{\alpha}{\alpha+3 \lambda}\right)^{\frac{n}{2}}$. Defining $\overline{B_{\alpha}(f)}=\frac{B_{\alpha}(f)}{\sqrt{C^{\prime}}\left(\frac{\alpha}{\alpha+\lambda}\right)^{\frac{n}{4}}}$ and repeating calculations we find the result. 


\section{Proof of Theorem 1.3}

Before starting to let us fix some notation, we consider a generic Gaussian state with $\psi_{\lambda}=K \mathrm{e}^{-\frac{\lambda}{4(1+\lambda)}(z+\bar{z})^{2}}$ where $K=\frac{1}{\sqrt{\mathrm{e}}}\left(\frac{\alpha}{\alpha+\lambda}\right)^{\frac{n}{2}}$ in dimension $n=1$. We denote by $\sigma_{\psi_{\lambda}}^{2}(x), \sigma_{\psi_{\lambda}}^{2}(p)$ respectively the variance of the observable $x$ and $p$. We have that:

$$
\sigma_{\psi_{\lambda}}^{2}(x)=\int_{-\infty}^{+\infty} x^{2} K^{2} \mathrm{e}^{-\frac{\lambda}{1+\lambda} x^{2}} \mathrm{~d} x=\frac{K^{2} \sqrt{\pi}(1+\lambda)^{\frac{3}{2}}}{2 \lambda^{\frac{3}{2}}}
$$

where for the evaluation the general result in one dimension is useful:

$$
\int_{-\infty}^{+\infty} x^{n} \mathrm{e}^{-a x^{2}} \mathrm{~d} x=\frac{1 \cdot 3 \cdot 5 \cdots(n-1) \sqrt{\pi}}{2^{\frac{n}{2}} a^{\frac{n+1}{2}}}, \quad n=2,4,6, \cdots, a>0 .
$$

Now we must evaluate $\sigma_{\psi_{\lambda}}^{2}(p)$. In a similar way as before we have:

$$
\begin{aligned}
\sigma_{\psi_{\lambda}}^{2}(p) & =\int_{-\infty}^{+\infty} K^{2}\left(\partial_{x} \mathrm{e}^{-\frac{\lambda}{2(1+\lambda)} x^{2}}\right)^{2} \mathrm{~d} x \\
& =K^{2} \frac{\lambda^{2}}{(1+\lambda)^{2}} \int_{-\infty}^{\infty} x^{2} \mathrm{e}^{-\frac{\lambda}{(1+\lambda)} x^{2}}=\frac{K^{2} \lambda^{2} \sqrt{\pi}(1+\lambda)^{\frac{3}{2}}}{2(1+\lambda)^{2} \lambda^{\frac{3}{2}}} .
\end{aligned}
$$

In order to prove the Heisenberg principle we must evaluate:

$$
\frac{1}{4}\left(K^{2} \int_{-\infty}^{+\infty} \mathrm{e}^{-\frac{\lambda}{1+\lambda} x^{2}} \mathrm{~d} x\right)^{2}=\frac{K^{4} \pi(1+\lambda)}{4 \lambda} .
$$

Now by the ordinary Heisenberg principle:

$$
\sigma_{\psi_{\lambda}}^{2}(x) \cdot \sigma_{\psi_{\lambda}}^{2}(p) \geq \frac{1}{4}\left(K^{2} \int_{-\infty}^{+\infty} \mathrm{e}^{-\frac{\lambda}{1+\lambda} x^{2}} \mathrm{~d} x\right)^{2}
$$

and substituting our quantities we find that:

$$
\frac{1+\lambda}{\lambda} \geq \frac{1+\lambda}{\lambda},
$$

this is true for every compression $\lambda>0$.

\section{Conflicts of Interest}

The author declares no conflicts of interest regarding the publication of this paper.

\section{References}

[1] Berezin, F.A. (1975) General Concept of Quantization. Communications in Mathematical Physics, 40, 153-174. https://doi.org/10.1007/BF01609397

[2] Engliš, M. (2016) An Excursion into Berezin-Toeplitz Quantization and Related Topics. In: Bahns, D., Bauer, W. and Witt, I., Eds., Quantization, PDEs, and Geo- 
metry. Operator Theory: Advances and Applications, 251, 69-115. https://doi.org/10.1007/978-3-319-22407-7_2

[3] Engliš, M. (2006) Berezin and Berezin-Toeplitz Quantizations for General Function Spaces. Revista Matemática Complutense, 19, 385-430.

[4] Ali, S.T. and Engliš, M. (2005) Quantization Methods: A Guide for Physicists and Amalysts. Reviews in Mathematical Physics, 17, 391-490. https://doi.org/10.1142/S0129055X05002376

[5] Berezin, F.A. and Šhubin, M.A. (1970) Symbols of Operators and Quantization. Proceedings of the Colloquia Math. Soc. Janos Bolyai, Tihany, 1970, 21-52.

[6] Griffiths, D.J. (2004) Introduction to Quantum Mechanics. 2nd Edition, Pearson.

[7] Hall, B.C. (2013) Quantum Theory for Mathematicians. Springer, Graduate Texts in Mathematics.

[8] Stroethoff, K. (1997) The Berezin Transform and Operators on Spaces of Analytic Functions. Linear Operators Banach Center Publications, Institute of Mathematics Polish Academy of Sciences, Warszawa, Vol. 38.

[9] Zhu, K. (2015) Uncertainty Principles for the Fock Space. Preprint.

[10] Weyl, H. (1950) Gruppentheorie und Quantenmechanik. S. Hirzel, Leipzig, 1928. Revised English Edition: "The Theory of Groups and Quantum Mechanics", Methuen, London, 1931, Reprinted by Dover, New York, 1950. 